\title{
VALOR COALICIONAL Y ESTRATEGIAS PARLAMENTARIAS
}

\section{Francesc Carreras* y Guillermo Owen**}

\section{INTRODUCCION}

Iniciada a mediados de este siglo, la Teoría de Juegos ha venido esforzándose por reducir sensiblemente el mutuo alejamiento al que parecían estar condenadas las Matemáticas y algunas de las denominadas Ciencias Sociales, en particular la Ciencia Política. La Teoría de Juegos proporciona nuevas ideas para el tratamiento de situaciones en que convergen y/o se enfrentan diversos intereses. La Ciencia Política abre un campo de interpretación natural para la aplicación fecunda de estas ideas.

El objetivo no es reducir a números la complejidad y sutileza de las relaciones políticas. Ni tratar de predecir el comportamiento de individuos y grupos influenciados a menudo por circunstancias que los modelos formales - pese a su creciente sofisticación - no llegan a incorporar. Se pretende, simplemente, aportar una componente de racionalidad matemática a una sección importante del análisis político. El contraste de los resultados teóricos con la realidad ha de ser - haya coincidencia o no- necesariamente instructivo.

En este trabajo proponemos la aplicación de conceptos y técnicas de la teoría de juegos cooperativos al análisis del comportamiento estratégico de los

* Departamento de Matemática Aplicada II, Universidad Politécnica de Cataluña.

** Naval Postgraduate School, Monterey, California. 
partidos en el ámbito parlamentario. La finalidad principal es el estudio de la formación de coaliciones, ya sean éstas de gobierno o de apoyo político.

A modo de ilustración, incluimos dos ejemplos a los que hemos dedicado nuestra atención en el pasado: en la sección 2, el Parlamento de Cataluña, Legislatura 1980-1984 [cf. Carreras y Owen (1988)]; en la sección 4, el Parlamento del País Vasco, Legislatura 1986-1990 [Carreras y Owen (1995)]. Las diferencias de tratamiento se justifican por las circunstancias que rodeaban el comienzo de una y otra legislatura, y la sección 3 aporta la inflexión teórica necesaria. Un estudio similar referido a las trece Comunidades Autónomas de Régimen Común puede verse en Carreras, García-Jurado y Pacios (1993).

Para la representación de las estructuras de decisión colectiva de carácter parlamentario utilizaremos juegos simples y, en particular, juegos de mayoría ponderada. Un elemento de juicio interesante como punto de partida es la distribución del poder de negociación entre los partidos. Para este fin hemos elegido el valor de Shapley para juegos cooperativos [Shapley (1953)], cuya restricción a los juegos simples se conoce a veces con el nombre de índice de poder de Shapley-Shubik [cf. Shapley y Shubik (1954)]. El valor suministrará las condiciones iniciales para el regateo coalicional.

Sin embargo, la formación de coaliciones altera, por lo general, la distribución inicial de poder. Es conveniente, por tanto, disponer de una medida complementaria capaz de evolucionar sincrónicamente con la dinámica coalicional y, en particular, reflejar los estados finales a los que pueden conducir las negociaciones.

El valor coalicional [Owen (1977)] se adapta a estos propósitos. Se trata de una generalización natural del valor de Shapley que nos devuelve al mismo si los jugadores llegan a formar la coalición total, condiciones que implican una perfecta coherencia o "continuidad». El valor coalicional es una medida de la distribución de poder que se ajusta a cada posible estructura de coaliciones, valorando los efectos de su formación a tres niveles: valor obtenido por cada coalición al constituirse efectivamente, distribución de dicho valor entre los miembros de la misma y valor residual que reciben los jugadores que no se han integrado en ninguna de las coaliciones formadas. Por tanto, permite a cada partido evaluar su posición estratégica no sólo dentro de la coalición en la que se ha integrado, sino también relativamente a otras coaliciones que hayan podido formarse.

El programa puede concretarse en dos puntos: seleccionar qué coaliciones deberían formarse y fundamentar el criterio por el que se lleva a cabo esta selección. Entre los factores relevantes que promueven la constitución de una coalición destacan su capacidad para proporcionar una posición aceptable a cada uno de sus integrantes y sus expectativas de estabilidad como ente colectivo. Además, los beneficios alcanzados por una coalición deberían ser susceptibles de repartirse entre sus miembros en función de la contribución de cada uno al proyecto común.

En estas condiciones, la existencia de coaliciones que optimicen el valor coa- 
licional de cada uno de sus miembros asegura a éstos plena satisfacción y, por ello mismo, garantiza la estabilidad del compromiso acordado. Esta parte final del análisis adopta un carácter no cooperativo. Cada partido pretende optimizar su valor coalicional, indicador de su cuota de poder según el escenario. La estabilidad coalicional corresponde a la idea de equilibrio fuerte de Nash en un juego no cooperativo en forma normal, ya que ningún miembro o subcoalición tiene incentivos para romper el acuerdo. El problema fundamental es que no siempre existen coaliciones estables en este sentido.

Un punto crucial, al que dedicaremos la sección 3, es la influencia que las restricciones de carácter ideológico o táctico pueden ejercer sobre las posibilidades de cooperación y, por tanto, en la distribución de poder y en el resultado final de la negociación. Pondremos de manifiesto que es factible incorporar al análisis restricciones de dos tipos: de afinidad, generalizando la idea de conexión ideológica en un espectro unidimensional [Axelrod (1970)], y de incompatibilidad, más radical que el anterior. En ambos casos las restricciones estarán definidas por grafos — relaciones binarias irreflexivas y simétricas—, y esta información de origen externo quedará incorporada modificando adecuadamente la estructura formal original. La referencia básica para esta cuestión es Carreras (1991), aunque al final de la sección 3 mencionaremos varias generalizaciones obtenidas recientemente.

Adoptaremos como hipótesis de trabajo los siguientes supuestos habituales: 1) Se asume una perfecta disciplina de voto dentro de cada grupo parlamentario, lo que permite identificar a los partidos políticos como los agentes del juego coalicional. La excepción obvia es el eventual grupo mixto, que se supondría descompuesto en subgrupos ideológicamente homogéneos. 2) Las decisiones del cuerpo colegiado son del tipo binario, en el que una propuesta se enfrenta al status quo. 3) Se exige mayoría absoluta para la aprobación, entendiendo que la ausencia sistemática de un partido se traduce en el correspondiente descenso de la mayoría. La teoría es aplicable, no obstante, a situaciones regidas por mayorías cualificadas. 4) Los partidos tienden a formar coaliciones ganadoras minimales en caso de que ninguno de ellos alcance la mayoría exigida. 5) La utilidad del poder, y por tanto del valor coalicional, es fraccionable y transferible.

\section{EL PARLAMENTO DE CATALUÑA, LEGISLATURA 1980-1984}

Como primera aplicación de la teoría del valor coalicional al análisis de las estrategias parlamentarias, consideraremos en esta sección la situación coalicional en el Parlamento de Cataluña tras las elecciones celebradas el 30 de marzo de 1980.

Después de la muerte de Franco, en 1975, España se convirtió en una monarquía parlamentaria, protagonizando una transición que ha sido considerada modélica en muchos sentidos. Las elecciones generales de 1977 conduje- 
ron a la formación de un gobierno minoritario de UCD, sustentado por los 165 representantes de este grupo en un Congreso de los Diputados de 350 escaños. Pacte Democràtic per Catalunya, germen de la futura CiU, obtuvo 11 escaños.

Tras la aprobación en 1978 de la Constitución, que dio vía libre al que daría en llamarse el "Estado de las Autonomías», las segundas elecciones generales, en 1979, dieron de nuevo la victoria por mayoría relativa a UCD, esta vez con 168 escaños. CiU alcanzó 8 escaños. En 1980, tres semanas antes que Cataluña, también el País Vasco celebró sus primeras elecciones autonómicas.

El marco ideológico y estratégico de la joven partitocracia instaurada en España era singular. Lo describe Jordi Capo en un detenido y lúcido análisis [Capo (1986)] del que extraemos, muy resumidos, los aspectos principales. Tras una primera etapa desarrollada en un clima de consenso generalizado, que se concreta en los Pactos de la Moncloa — de carácter político, económico y social- y la aprobación de la Constitución, se pasa a un ambiente de confrontación propiciado por las elecciones generales de marzo de 1979 y, sobre todo, por las municipales del mes siguiente, que dan la victoria al PSOE, el PCE y los partidos regionalistas. UCD gobierna en solitario el país desde Madrid, alcanzando pactos puntuales con las distintas fuerzas.

Los partidos políticos carecen aún de experiencia, ofrecen en general una estructura cambiente y sin cohesión interna, desconocen su verdadero soporte sociológico entre el electorado y no se han situado definitivamente en el espectro ideológico, un espectro complejo que no se reduce al clásico eje izquierdaderecha, sino que depende de las relaciones centro-periferia e incluso de los orígenes históricos. UCD acabará por sucumbir a la heterogeneidad de su procedencia y de sus aspiraciones. Enfrente, el PSOE, un grupo homogéneo con estructura federal, programa, tradición y perspectivas de crecimiento rápido, se prepara para tomar la alternativa.

Las coaliciones — si de ellas puede hablarse en esta época- no se ajustan a la teoría formal. El primer período da cabida a acuerdos muy globales, contribuyendo a la conexión ideológica la postura centrada de las dos grandes formaciones, UCD y PSOE, que aspiran a un bipartidismo estable. En el segundo período, sin embargo, los partidos menores asumen un papel significativo. UCD alcanza pactos puntuales con algunos de ellos en función de cuál de sus tendencias domina su discusión interna, mientras, a nivel local, es el PSOE quien suele establecer acuerdos con el PCE y/o las fuerzas regionales para asegurarse el control. El inicio de la serie de elecciones autonómicas contribuye a aumentar esta efervescencia. La "cultura de la coalición» comienza a arraigar muy lentamente en España.

En estas circunstancias y con una participación del 61 por 100 sobre un censo de 4.385.190 votantes, las elecciones del 30 de marzo de 1980 atribuyeron los 135 escaños del Parlamento de Cataluña del siguiente modo: 


\begin{tabular}{|c|c|}
\hline Partido & Escaños \\
\hline $\mathrm{CiU}$ (Convergència i Unió) & 43 \\
\hline PSC (Partit dels Socialistes de Catalunya - PSOE) .......... & 33 \\
\hline PSUC (Partit Socialista Unificat de Catalunya - PCE) ... & 25 \\
\hline 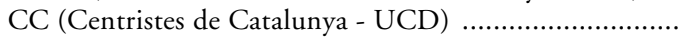 & 18 \\
\hline 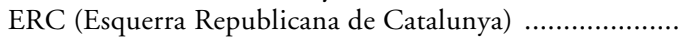 & 14 \\
\hline 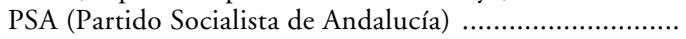 & 2 \\
\hline
\end{tabular}

Puesto que las votaciones se rigen — salvo situaciones excepcionales- por mayoría absoluta (68/135), la estructura formal del Parlamento queda descrita por el juego de mayoría ponderada

$$
[68 ; 43,33,25,18,14,2],
$$

es decir, por el juego simple $(N, W)$, donde

$$
N=\{\mathrm{CiU}, \mathrm{PSC}, \mathrm{PSUC}, \mathrm{CC}, \mathrm{ERC}, \mathrm{PSA}\}
$$

y la familia de las coaliciones ganadoras $W$ está formada por aquellas coaliciones que controlan 68 votos o más. No es difícil calcular el valor de Shapley de este juego, que queda reflejado en la segunda columna numérica de la tabla 1.

\begin{tabular}{|c|c|c|c|c|}
\hline Partido & Escaños & Valor & $P S C+P S U C$ & $C i U+C C$ \\
\hline $\mathrm{CiU}$. & 0,3185 & 0,4000 & 0,1667 & 0,4167 \\
\hline PSC & 0,2444 & 0,2333 & 0,2500 & 0,1667 \\
\hline 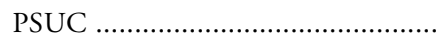 & 0,1852 & 0,2333 & 0,2500 & 0,1667 \\
\hline 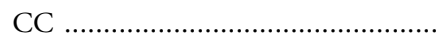 & 0,1333 & 0,0667 & 0,1667 & 0,0833 \\
\hline ERC & 0,1037 & 0,0667 & 0,1667 & 0,1667 \\
\hline PSA & 0,0148 & 0 & & \\
\hline
\end{tabular}

\section{TABLA 1}

Como se observa al comparar con la primera columna, que nos indica la fracción de escaños ocupados por cada partido, CC y ERC, con el 13,33 y el 10,37 por 100 de escaños, respectivamente, tienen un poder considerablemente menor, del 6,67 por 100. En cambio, CiU y PSUC están en mejor posición estratégica que la que indican sus escaños (el segundo llega a equipararse con el PSC), mientras que el PSA es un jugador nulo, es decir, sin valor estratégico.

Introduciremos a continuación consideraciones de tipo coalicional, describiendo en cada caso el correspondiente efecto sobre la distribución de poder. 
Por una parte, parece natural considerar una coalición entre los dos partidos principales de izquierda, PSC y PSUC, lo que daría la estructura de coaliciones

$$
\text { PSC }+ \text { PSUC }=\{\mathrm{CiU},\{\text { PSC, PSUC }\}, \mathrm{CC}, \mathrm{ERC}\}
$$

cuyo valor coalicional se indica en la tabla 1 , tercera columna.

Por otra parte, también $\mathrm{CiU}$ dispone de un compañero natural de coalición, CC. Esto lleva a la estructura

$$
\mathrm{CiU}+\mathrm{CC}=\{\{\mathrm{CiU}, \mathrm{CC}\}, \mathrm{PSC}, \mathrm{PSUC}, \mathrm{ERC}\}
$$

cuyo valor coalicional puede verse en la última columna de la tabla 1 .

Por descontado, es plausible suponer formadas simultáneamente ambas protocoaliciones: el valor coalicional correspondiente a la estructura

$$
\text { PSC + PSUC; CiU + CC }=\{\{C i U, C C\},\{P S C, P S U C\}, E R C\}
$$

figura en la primera columna de la tabla 2 .

En este punto, ERC se encuentra en una posición envidiable, puesto que con tan sólo 14 diputados (apenas un 10 por 100) tiene la clave de la situación. Puede, en efecto, abstenerse de entrar en coalición, reservando para situaciones puntuales su apoyo a un eventual gobierno minoritario de uno u otro signo. O determinar si Cataluña va a tener un gobierno de centro-derecha o bien de centro-izquierda, con mayoría parlamentaria en ambos casos. De decidirse por una de estas dos últimas opciones, la otra protocoalición se disolvería con toda probabilidad.

Si ERC se une a la coalición de centro-derecha se obtiene la estructura

$$
(\mathrm{CiU}+\mathrm{CC})+\mathrm{ERC}=\{\{\{\mathrm{CiU}, \mathrm{CC}\}, \mathrm{ERC}\}, \mathrm{PSC}, \mathrm{PSUC}\}
$$

y en el otro caso

$$
(\mathrm{PSC}+\mathrm{PSUC})+\mathrm{ERC}=\{\mathrm{CiU},\{\{\mathrm{PSC}, \mathrm{PSUC}\}, \mathrm{ERC}\}, \mathrm{CC}\}
$$

\begin{tabular}{|c|c|c|c|}
\hline Partido & $\begin{array}{c}P S C+P S U C \\
C i U+C C\end{array}$ & $(C i U+C C)+E R C$ & $(P S C+P S U C)+E R C$ \\
\hline 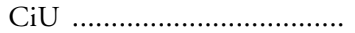 & 0,1667 & 0,5000 & 0 \\
\hline 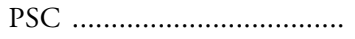 & 0,1667 & 0 & 0,3333 \\
\hline 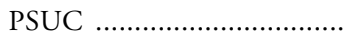 & 0,1667 & 0 & 0,3333 \\
\hline CC & 0,1667 & 0,1667 & 0 \\
\hline 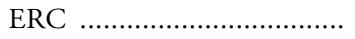 & 0,3333 & 0,3333 & 0,3333 \\
\hline
\end{tabular}

Los valores coalicionales correspondientes se dan en la tabla 2, segunda y tercera columnas, respectivamente.

TABLA 2 
En cualquiera de las tres situaciones contempladas en la tabla 2 la posición de ERC es igualmente satisfactoria, y la decisión final quedaría, desde este punto de vista, a merced de las preferencias ideológicas o estratégicas de esta formación. En ERC concurren dos factores predominantes: una filosofía de izquierdas y un nacionalismo radical. Este último tuvo, quizás, la influencia decisiva, porque ERC se decidió por la protocoalición de centro-derecha, constituyéndose lo que llegó a ser conocido como el "rodillo parlamentario»: la coalición $\{\{\mathrm{CiU}, \mathrm{CC}\}, \mathrm{ERC}\}$. Extrañamente (no tanto según nuestro análisis), el partido menor, con 14 escaños, acabó por resolver la tensión estratégica y dio una cómoda mayoría al gobierno de la Generalitat.

En cuanto a las cifras atribuidas por el valor coalicional a cada uno de los elementos de la coalición gobernante, éstas se ajustan satisfactoriamente a la distribución real de los cargos principales. En efecto, ERC obtuvo la Presidencia del Parlamento y una conselleria, mientras que CiU ocupó todos los demás puestos, incluyendo, como es lógico, la Presidencia de la Generalitat. El papel secundario de CC puede explicarse a cuenta de cierto apoyo de CiU al gobierno de UCD en Madrid (recuérdese la abstención de CiU en la moción de censura contra Adolfo Suárez en mayo de 1980 y su votación a favor de la investidura de Leopoldo Calvo Sotelo en febrero de 1981).

Como es conocido, a mediados de la legislatura comenzaron a producirse cambios. El PSA se disolvió y tanto el PSUC como CC sufrieron escisiones, dando lugar, respectivamente, al PCC y el CDS. También ERC experimentó tensiones internas y pagó, quizás, electoralmente, su intervención en la coalición de gobierno, ya que en las tres legislaturas siguientes, ganadas por $\mathrm{CiU}$ con mayoría absoluta, ERC obtuvo 5, 6 y 11 escaños.

Nuestro estudio del Parlamento de Cataluña ha consistido en evaluar diversas estructuras de coaliciones plausibles políticamente según criterios de conexión ideológica. La evolución del valor coalicional pone de manifiesto la tensión creada por las estrategias que conducen a la formación de coaliciones conexas minoritarias. En el punto culminante, la decisión de ERC, enfrentada a tres posibles salidas que optimizan su valor coalicional, se explica más por su carácter nacionalista y su voluntad de dar estabilidad al gobierno regional que por su mera posición en un eje ideológico convencional izquierda-derecha.

\section{RESTRICCIONES A LA COOPERACION}

El análisis efectuado en la sección anterior se ha fundado en el juego de mayoría ponderada que describía la estructura formal del Parlamento de Cataluña. Es cierto que hemos tenido en cuenta la conexión ideológica, pero sólo para seleccionar las (estructuras de) coaliciones plausibles y calcular el valor coalicional asociado. El punto de referencia ha sido en todo caso la estructura formal. 
Sin embargo, la situación de los partidos en el espectro ideológico acaba por influir en su comportamiento estratégico, asumiendo un papel sustancial, si no decisivo, durante la negociación coalicional intraparlamentaria. En esta sección resumimos los resultados acerca de esta cuestión obtenidos en un estudio al que remitimos al lector interesado en una exposición detenida [Carreras (1991)]. En la sección siguiente aplicaremos estas ideas a una situación real.

La manera natural de introducir información adicional en una situación dada es la modificación del juego en un sentido restrictivo, pasando de la estructura formal, dada por el juego inicial, a la estructura modificada, dada por el juego restringido. Por coherencia, al aplicar al juego restringido las dos medidas básicas de poder que han sido utilizadas hasta ahora, el valor de Shapley y el valor coalicional, nos referiremos a ellas como los valores modificados del juego original.

Mencionaremos dos métodos de restricción de juegos simples. En el primero de ellos se supone dado un grafo que representa un conjunto de relaciones de afinidad de tipo binario, no necesariamente transitivo. Estas afinidades pueden tener un origen ideológico, de forma que el modelo generaliza ampliamente la noción de conexión ideológica unidimensional clásica. El segundo procedimiento, más radical, parte de un grafo que representa un conjunto de relaciones de incompatibilidad. Los argumentos en favor de la racionalidad del proceso de restricción en ambos casos son semejantes, pero es necesario remarcar que los dos métodos no son equivalentes, sino más bien complementarios. Buena prueba de ello es que, bajo una elemental condición de consistencia, es factible aplicarlos simultáneamente.

Antes de proceder a la descripción de los dos métodos será conveniente hacer algunas precisiones sobre notación y nomenclatura. Un grafo en un conjunto $N$ es una colección $J$ de subconjuntos de dos elementos de $N$, denotados ijj y llamados aristas del grafo. Un camino en un subconjunto $S \subseteq N$ es una serie $i_{1}, i_{2}, \ldots, i_{k}$ de elementos de $S$ tales que cada dos consecutivos forman una arista. Un subconjunto $S$ es conexo si para cada par $i, j \in S, i \neq j$, existe un camino en $S$ tal que $i=i_{1}, j=i_{k}$. Un subconjunto $S$ es admisible si ningún par de sus elementos está unido por una arista del grafo.

Sea $(N, W)$ un juego simple arbitrario y sea $A$ un grafo en $N$, interpretado como conjunto de relaciones de afinidad entre los jugadores. Se define

$$
W^{A}=\left\{S \subseteq N: S_{0} \subseteq S \text { para alguna } S_{0} \in W \text { y } A-\text { conexa }\right\}
$$

y se denomina a $\left(N, W^{A}\right)$ el juego restringido por el grafo de afinidades $A$. $\left(N, W^{A}\right)$ es un juego simple, que hereda de $(N, W)$ las propiedades de monotonía y superaditividad. En cambio, no es extraño que deje de ser un juego de mayoría ponderada aun siéndolo $(N, W)$. Por último, dado que $W^{A} \subseteq W$, es habitual que el juego restringido sea menos decisivo — más fácil de bloquearque el juego original, algo natural desde el momento en que introducimos dificultades para la formación de coaliciones. 
Remitimos al lector a la referencia citada al principio de esta sección para una discusión detallada de otras posibilidades de definición del juego restringido. Allí encontrará los argumentos que sustentan la opción escogida, consideraciones sobre el cálculo del valor de Shapley de $\left(N, W^{A}\right)$ y la forma de obtener $\left(W^{A}\right)^{m}$ directamente a partir de $W^{m}$ y, finalmente, las relaciones de este método con la idea de comunicación restringida elaborada por Myerson (1977) — a partir de supuestos diferentes - para juegos cooperativos en general [cf. también Owen (1986)].

Tomemos ahora de nuevo un juego simple arbitrario $(N, W)$ y sea $I$ un grafo en $N$ interpretado como un conjunto de incompatibilidades entre jugadores. Definimos

$$
W^{I}=\left\{S \subseteq N: S_{0} \subseteq S \text { para alguna } S_{0} \in W \text { e } I \text {-admisible }\right\}
$$

y llamamos a $\left(N, W^{I}\right)$ el juego restringido por el grafo de incompatibilidad $I$. Todos los comentarios relativos al primer procedimiento son, mutatis mutandis, aplicables aquí, con los añadidos siguientes.

En primer lugar, no existen precedentes en la literatura de la teoría de juegos acerca de esta idea. Por otra parte, al ser hereditaria la condición de admisibilidad, el cálculo de las coaliciones ganadoras minimales del juego restringido es aquí directo, ya que

$$
\left(W^{I}\right)^{m}=\left\{S \in W^{m}: S \text { es } I-\text { admisible }\right\}
$$

Nuestro último comentario se refiere a la posibilidad de considerar simultáneamente un grafo de afinidades $A$ y un grafo de incompatibilidades $I$ afectando a un juego $(N, W)$. Para elaborar una teoría coherente basta con exigir la condición de consistencia

$$
A \cap I=\varnothing,
$$

que impide la presencia de pares de jugadores afines e incompatibles a la vez. Bajo este supuesto, la definición natural de juego restringido por $A$ e $I$ será la dada por

$$
W^{A I}=\left\{S \subseteq N: S_{0} \subseteq S \text { siendo } S_{0} \in W, A \text { - conexa e } I \text {-admisible }\right\}
$$

Debemos mencionar diversos trabajos que se han ocupado de generalizar estas ideas. Bergantiños et al. (1993) extienden la restricción por incompatibilidades (o por afinidades e incompatibilidades dadas consistentemente) a los juegos cooperativos en general. Vázquez-Brage et al. (1995) trasladan la teoría del valor coalicional a juegos cooperativos afectados por una relación de afinidad. Finalmente, Amer y Carreras (1995a) y (1995b) generalizan drásticamente - también para juegos cooperativos en general- el concepto de coopera- 
ción restringida, situándolo en un nivel cuantitativo, y por tanto mucho más preciso, mediante los indices de cooperación (hipergrafos ponderados). De este modo definen el marco más general para el estudio de la cooperación restringida alcanzado hasta la fecha.

\section{EL PARLAMENTO DEL PAIS VASCO, LEGISLATURA 1986-1990}

En esta sección combinaremos la teoría del valor coalicional con la técnica de restricción de juegos por incompatibilidades, y examinaremos el caso del Parlamento vasco después de las elecciones celebradas el 30 de noviembre de 1986.

Prosiguiendo con la reseña histórica en el punto donde la habíamos abandonado en la sección 2, en las primeras elecciones autonómicas vascas salió vencedor el PNV, con 25 escaños sobre 60. La sistemática inasistencia de los 11 diputados de HB permitió al PNV gobernar con comodidad en solitario durante cuatro años. Las otras dos comunidades históricas inauguraban también sus parlamentos autonómicos, Galicia en 1981 y Andalucía en 1982. La mayoría absoluta conseguida por el PSOE en esta última región fue un presagio de su gran triunfo en las legislativas celebradas dentro de ese mismo año. Los socialistas se impusieron de nuevo en 1983 con motivo de las primeras elecciones autonómicas en las trece Comunidades de Régimen Común. Alcanzaron mayoría absoluta en ocho de ellas y mayoría relativa en otras tres, empatando en número de escaños en Castilla y León con una titubeante AP que, aun siendo el primer partido de la oposición en el Congreso de los Diputados, sólo dominaba Cantabria por mayoría absoluta y Galicia sin ella.

En 1984 se amplió a 75 el número de escaños del Parlamento vasco, y el PNV obtuvo 32 diputados, que dada la inasistencia de los 11 representantes de $\mathrm{HB}$, le servían para gobernar "casi» con mayoría absoluta. Mientras, en Cataluña, CiU obtenía mayoría absoluta, y hacían lo propio AP en Galicia (1985) y el PSOE en Andalucía (1986), después de haber barrido de nuevo los socialistas tanto en las elecciones al Parlamento Europeo, con motivo del ingreso de España y Portugal en la Comunidad, como en las generales de ese mismo año.

Entretanto, el PNV, además de ver reducidos de 8 a 6 sus representantes en el Congreso de Diputados de 1986, experimentaba una gravísima crisis interna, que desembocaría en la dimisión del hasta entonces lehendakari, Carlos Garaicoechea (sustituido por José Antonio Ardanza), y su salida del partido para crear con sus seguidores una nueva formación, EA. La convocatoria de elecciones anticipadas en el País Vasco era inevitable, e iban a participar en ellas un partido socialista en auge, un nacionalismo vasco muy disgregado y un centro-derecha con poca fuerza. Estas circunstancias propiciaron el primer registro serio de la "cultura de la coalición», hasta entonces casi inexistente en España y extendida posteriormente a otros ámbitos.

El estudio que presentamos fue realizado entre el 30 de noviembre de 1986 
y el 21 de enero de 1987. Durante este tiempo, e incluso después, los partidos estuvieron negociando para formar una coalición, ya que ninguno de ellos había obtenido la mayoría absoluta. Las dudas acerca de si HB mantendría su «tradicional» postura de inasistencia complicaron un poco más el análisis.

Con una participación del 70 por 100 sobre un censo de 1.650 .686 votantes, las elecciones del 30 de noviembre de 1986 distribuyeron los 75 escaños del Parlamento del País Vasco como sigue:

\begin{tabular}{|c|c|}
\hline Partido & Escaños \\
\hline PSE (Partido Socialista de Euskadi - PSOE) .. & 19 \\
\hline PNV (Partido Nacionalista Vasco) ............... & 17 \\
\hline 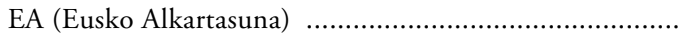 & 13 \\
\hline HB (Herri Batasuna) ........ & 13 \\
\hline EE (Euskadiko Ezkerra) ..... & 9 \\
\hline CP (Coalición Popular) ... & 2 \\
\hline CDS (Centro Democrático y Social) .................................. & 2 \\
\hline
\end{tabular}

Puesto que la mayoría absoluta es en este caso 38, la estructura formal del Parlamento vasco es el juego de mayoría ponderada

$$
[38 ; 19,17,13,13,9,2,2] \text {, }
$$

cuyo conjunto de jugadores es

$$
N=\{\mathrm{PSE}, \mathrm{PNV}, \mathrm{EA}, \mathrm{HB}, \mathrm{EE}, \mathrm{CP}, \mathrm{CDS}\}
$$

Las coaliciones ganadoras minimales son las siguientes:

$\{$ PSE, PNV, EA
$\{$ PSE, PNV, HB $\}$
$\{$ PSE, PNV, EE
$\{$ PSE, PNV, CP $\}$
$\{$ PSE, PNV, CDS
$\{$ PSE, EA,$H B\}$

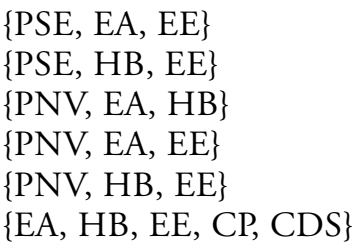

El valor de Shapley de este juego queda reflejado en la segunda columna de la tabla 3. Es interesante señalar que, a pesar de las diferencias en el número de escaños (véase la primera columna), el PSE y el PNV, por un lado, y EA, HB y EE, por otro, disponen de la misma capacidad estratégica, según indica el valor. Estas equivalencias de posiciones pueden apreciarse también inspeccionando con alguna atención la anterior lista de coaliciones ganadoras minimales. 


\section{TABLA 3}

\begin{tabular}{|c|c|c|c|}
\hline Partido & Escaños & Valor formal & Modificado \\
\hline PSE & 0,2533 & 0,2524 & 0,2333 \\
\hline 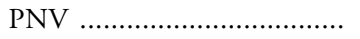 & 0,2267 & 0,2524 & 0,3167 \\
\hline 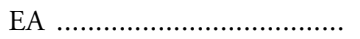 & 0,1733 & 0,1524 & 0,2333 \\
\hline 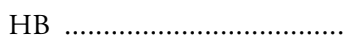 & 0,1733 & 0,1524 & 0,0333 \\
\hline 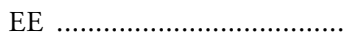 & 0,1200 & 0,1524 & 0,1500 \\
\hline 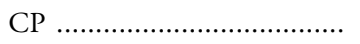 & 0,0267 & 0,0190 & 0 \\
\hline CDS & 0,0267 & 0,0190 & 0,0333 \\
\hline
\end{tabular}

Nuestro objetivo es la evaluación de las coaliciones ganadoras minimales y el análisis comparativo de los resultados obtenidos. Sin embargo, el valor coalicional es la solución a un regateo interno en cada coalición en el que cada jugador que se dispone a entrar en ella aduce las posibilidades de intervenir en coaliciones con jugadores externos a las que ha renunciado - y esta sensibilidad es una de las principales virtudes del valor coalicional-. Por tanto, las coaliciones impensables políticamente no sólo no merecen ser evaluadas, sino que carecen de valor como argumentos para la negociación. Esto implica que el punto de partida para el estudio de la formación de coaliciones no debe ser la estructura formal, sino la estructura modificada.

Esencialmente, se trata de asignar probabilidad cero a la formación de coaliciones que sean inaceptables desde el punto de vista ideológico o coyuntural. Esta es una tarea delicada para el observador, porque la declaración transparente de las propias preferencias no es una de las tácticas habituales en la negociación política. En nuestro caso tomamos en consideración: 1) el comportamiento previo de los partidos; 2) las manifestaciones efectuadas por sus líderes y portavoces a los medios de comunicación durante el período de negociación. El tipo de relaciones entre los partidos objeto de estudio sugirió que el segundo de los métodos de restricción presentados en la sección anterior era el más adecuado en este caso.

A partir de la relación de incompatibilidad binaria que afecta a los pares

$$
\text { PSE/HB, PSE/CP, HB/EE, HB/CP, }
$$

asignamos probabilidad nula a cualquier coalición que contenga a alguno de estos pares. Las coaliciones ganadoras minimales que superan este test de admisibilidad son

\{PSE, PNV, EA\}

\{PSE, PNV, EE\}

\{PSE, PNV, CDS $\}$
\{PSE, EA, EE\}

$\{\mathrm{PNV}, \mathrm{EA}, \mathrm{HB}\}$

$\{\mathrm{PNV}, \mathrm{EA}, \mathrm{EE}\}$ 
y definen en $N$ el juego restringido que nos da la estructura modificada del Parlamento vasco.

El valor de Shapley del juego restringido aparece en la última columna de la tabla 3, y se observan variaciones notables respecto a la columna anterior. El PNV es ahora el partido principal; el PSE ha sido desplazado a una segunda posición, que comparte con EA; EE se sostiene y, en cambio, HB ha experimentado el mayor retroceso, quedando con el mismo valor que el CDS. Por su parte, CP pasa a ser jugador nulo. Las equivalencias de posición que sugiere el valor de Shapley corresponden al hecho de que la permutación que intercambia PSE por EA y HB por CDS deja invariante el juego restringido (se dice que la permutación es un automorfismo de este juego).

La estructura modificada será el punto de partida de nuestro análisis coalicional y, siguiendo un criterio clásico [cf. Riker (1962)], evaluaremos exclusivamente las seis coaliciones ganadoras minimales. Si una de ellas se constituye controlará el cien por cien del poder, que será distribuido entre sus integrantes por el valor coalicional. Al ser ternarias, cada una de estas coaliciones admite cuatro vías de formación, que por lo general reciben tratamientos diferentes al efectuar el reparto en términos del valor coalicional. Si A, B y C son los partidos que van a formar coalición, representaremos los cuatro resultados posibles por

$$
\mathrm{A}+\mathrm{B}+\mathrm{C}, \quad(\mathrm{A}+\mathrm{B})+\mathrm{C}, \quad \mathrm{A}+(\mathrm{B}+\mathrm{C}), \quad(\mathrm{A}+\mathrm{C})+\mathrm{B} .
$$

El primero significa que los tres partidos se ponen de acuerdo simultáneamente, mientras que los demás corresponden a la formación de una protocoalición binaria a la que se une luego el tercer partido (hemos usado ya esta notación en el caso del Parlamento de Cataluña).

La tabla 4 muestra el resultado de nuestra evaluación, es decir, el valor coalicional que corresponde a cada partido según la coalición que se forma. En general, cada partido mejora, al entrar en una coalición, la utilidad que le asignaba inicialmente el valor de Shapley (tan sólo 3 de los 72 resultados se apartan de esta regla). En cambio, no hay reglas fijas en cuanto a la posición preferida por los partidos dentro de una coalición concreta. En la mitad de los casos, los tres partidos prefieren tomar la iniciativa e intervenir en la protocoalición o, si no, entrar simultáneamente, en tanto que en la otra mitad los partidos prefieren esperar a ser invitados y, en segundo término, entrar simultáneamente. Por último, las coaliciones del segundo bloque dan resultados análogos a los del sexto porque originan situaciones isomorfas según el automorfismo mencionado antes, y la misma relación existe entre los bloques tercero y quinto.

En este punto el análisis adopta un carácter no cooperativo. Cada partido localiza su posición óptima, es decir, la coalición y modo de formación (fila de la tabla 4) que le asigna el valor coalicional máximo. La selección se refleja en la tabla 5 . 


\section{TABLA 4}

\begin{tabular}{|c|c|c|c|c|c|c|}
\hline & PSE & $P N V$ & $E A$ & $H B$ & $E E$ & $C D S$ \\
\hline Valor de Shapley .................. & 0,2333 & 0,3167 & 0,2333 & 0,0333 & 0,1500 & 0,0333 \\
\hline $\mathrm{PSE}+\mathrm{PNV}+\mathrm{EA} \ldots \ldots \ldots \ldots \ldots$ & 0,3056 & 0,3889 & 0,3056 & & & \\
\hline$(\mathrm{PSE}+\mathrm{PNV})+\mathrm{EA} \ldots \ldots \ldots \ldots \ldots \ldots$ & 0,3750 & 0,4583 & 0,1667 & & & \\
\hline $\mathrm{PSE}+(\mathrm{PNV}+\mathrm{EA})$ & 0,1667 & 0,4583 & 0,3750 & & & \\
\hline$(\mathrm{PSE}+\mathrm{EA})+\mathrm{PNV} \ldots \ldots \ldots \ldots \ldots$ & 0,3750 & 0,2500 & 0,3750 & & & \\
\hline $\mathrm{PSE}+\mathrm{PNV}+\mathrm{EE} \ldots \ldots \ldots \ldots \ldots \ldots$ & 0,3056 & 0,4722 & & & 0,2222 & \\
\hline$(\mathrm{PSE}+\mathrm{PNV})+\mathrm{EE} \ldots \ldots \ldots \ldots \ldots$ & 0,3333 & 0,5000 & & & 0,1667 & \\
\hline $\mathrm{PSE}+(\mathrm{PNV}+\mathrm{EE})$ & 0,2500 & 0,5000 & & & 0,2500 & \\
\hline$(\mathrm{PSE}+\mathrm{EE})+\mathrm{PNV}$ & 0,3333 & 0,4167 & & & 0,2500 & \\
\hline $\mathrm{PSE}+\mathrm{PNV}+\mathrm{CDS} \ldots \ldots \ldots \ldots \ldots$ & 0,4028 & 0,4861 & & & & 0,1111 \\
\hline$(\mathrm{PSE}+\mathrm{PNV})+\mathrm{CDS} \ldots \ldots \ldots \ldots$ & 0,3750 & 0,4583 & & & & 0,1667 \\
\hline $\mathrm{PSE}+(\mathrm{PNV}+\mathrm{CDS}) \ldots \ldots \ldots \ldots . . . . .$. & 0,4583 & 0,4583 & & & & 0,0833 \\
\hline$(\mathrm{PSE}+\mathrm{CDS})+\mathrm{PNV} \ldots \ldots \ldots \ldots \ldots$ & 0,3750 & 0,5417 & & & & 0,0833 \\
\hline$P S E+E A+E E \ldots \ldots \ldots \ldots \ldots \ldots$ & 0,3889 & & 0,3889 & & 0,2222 & \\
\hline$(\mathrm{PSE}+\mathrm{EA})+\mathrm{EE}$ & 0,3750 & & 0,3750 & & 0,2500 & \\
\hline $\mathrm{PSE}+(\mathrm{EA}+\mathrm{EE})$ & 0,4167 & & 0,3750 & & 0,2083 & \\
\hline$(\mathrm{PSE}+\mathrm{EE})+\mathrm{EA}$ & 0,3750 & & 0,4167 & & 0,2083 & \\
\hline $\mathrm{PNV}+\mathrm{EA}+\mathrm{HB} \ldots \ldots \ldots \ldots \ldots$ & & 0,4861 & 0,4028 & 0,1111 & & \\
\hline$(\mathrm{PNV}+\mathrm{EA})+\mathrm{HB}$ & & 0,4583 & 0,3750 & 0,1667 & & \\
\hline $\mathrm{PNV}+(\mathrm{EA}+\mathrm{HB})$ & & 0,5417 & 0,3750 & 0,0833 & & \\
\hline$(\mathrm{PNV}+\mathrm{HB})+\mathrm{EA}$ & & 0,4583 & 0,4583 & 0,0833 & & \\
\hline $\mathrm{PNV}+\mathrm{EA}+\mathrm{EE} \ldots \ldots \ldots \ldots \ldots \ldots$ & & 0,4722 & 0,3056 & & 0,2222 & \\
\hline$(\mathrm{PNV}+\mathrm{EA})+\mathrm{EE} \ldots \ldots \ldots \ldots \ldots \ldots$ & & 0,5000 & 0,3333 & & 0,1667 & \\
\hline $\mathrm{PNV}+(\mathrm{EA}+\mathrm{EE}) \quad \ldots \ldots \ldots \ldots \ldots \ldots$ & & 0,4167 & 0,3333 & & 0,2500 & \\
\hline$(\mathrm{PNV}+\mathrm{EE})+\mathrm{EA} \ldots \ldots \ldots \ldots \ldots \ldots$ & & 0,5000 & 0,2500 & & 0,2500 & \\
\hline
\end{tabular}

\section{TABLA 5}

\begin{tabular}{lll}
\hline Partido & Coaliciones óptimas & Valor \\
\hline PSE & PSE+(PNV+CDS) & 0,4583 \\
PNV & (PSE+CDS)+PNV & 0,5417 \\
& PNV+(EA+HB) & 0,5417 \\
EA & (PNV+HB)+EA & 0,4583 \\
HB & (PNV+EA)+HB & 0,1667 \\
EE & PSE+(PNV+EE) & 0,2500 \\
& (PSE+EE)+PNV & 0,2500 \\
& (PSE+EA)+EE & 0,2500 \\
& PNV+(EA+EE) & 0,2500 \\
& (PNV+EE)+EA & 0,2500 \\
CDS & (PSE+PNV)+CDS & 0,1667
\end{tabular}


La posición de EE es singular, ya que en cuatro de sus cinco posibilidades ha de tomar la iniciativa e intervenir en la protocoalición binaria (EE fue, en realidad, uno de los partidos más interesados en la formación de una coalición). En cambio, los demás partidos hallan su mejor valor en coaliciones donde figuran como tercer socio, lo que, en principio, dificultaría cualquier acuerdo (y las negociaciones fueron, en efecto, lentas y complejas). Los bloques que destacan en la tabla 5 son $\{\mathrm{PSE}, \mathrm{PNV}, \mathrm{CDS}\}$ y $\{\mathrm{PNV}, \mathrm{EA}, \mathrm{HB}\}$, ya que cada uno de sus integrantes está interesado en alguna versión de los mismos.

Al reducir la discusión a los intervalos de valores que cada bloque ofrece a los partidos observamos que el bloque 1 no interesa a ninguno de sus componentes porque todos ellos se encuentran claramente mejor situados en otros bloques. Por idéntica razón, los bloques 2 y 6 carecen de atractivo para al menos uno de sus miembros. Estrictamente hablando, los bloques 3 y 5 disfrutan de una característica especial: atribuyen a cada uno de los partidos involucrados su mejor intervalo. Ello les proporciona, en caso de formarse, un alto grado de estabilidad, ya que ninguno de sus elementos tiene incentivos para abandonarlo. Por otra parte, dado que cada uno de los partidos preferiría aparecer como tercer socio, es plausible imaginar que se formaría la coalición paritaria.

En resumen, las coaliciones

\section{$\mathrm{PSE}+\mathrm{PNV}+\mathrm{CDS}, \quad \mathrm{PNV}+\mathrm{EA}+\mathrm{HB}$,}

que optimizan el valor coalicional de cada uno de sus miembros y por ello mismo gozan de estabilidad, pueden ser consideradas las soluciones racionales al problema de la negociación coalicional en el Parlamento vasco.

Volveremos a considerar este resultado después de discutir los efectos de la ausencia de HB. Como ya se ha señalado, en el momento de llevar a cabo este estudio no podía descartarse la posibilidad de que, al igual que en las dos legislaturas anteriores, HB decidiera mantenerse extramuros del Parlamento. Evaluaremos las consecuencias que se derivarían de esta decisión. La estructura formal del Parlamento vasco sin HB vendría dada por el juego de mayoría ponderada $[32 ; 19,17,13,9,2,2]$ relativo al conjunto de jugadores $N^{\prime}=\{\mathrm{PSE}, \mathrm{PNV}, \mathrm{EA}, \mathrm{EE}, \mathrm{CP}, \mathrm{CDS}\}$. Las coaliciones ganadoras minimales serían

$\begin{array}{ll}\{\mathrm{PSE}, \mathrm{PNV}\} & \{\text { PNV, EA, EE }\} \\ \{\mathrm{PSE}, \mathrm{EA}\} & \{\text { PNV, EA, CP }\} \\ \{\mathrm{PSE}, \mathrm{EE}, \mathrm{CP}, \mathrm{CDS}\} & \{\text { PNV, EA, CDS },\end{array}$

la única incompatibilidad a tener en cuenta sería PSE/CP y la estructura modificada del Parlamento estaría definida por las coaliciones ganadoras minimales que superan el test de admisibilidad, es decir, 

PSE, PNV\}
$\{\mathrm{PSE}, \mathrm{EA}\}$
$\{P N V, E A, E E\}$
$\{P N V, E A, C P\}$
$\{P N V, E A, C D S\}$

Los valores de Shapley formal y modificado se describen en la tabla 6, que el lector querrá probablemente analizar por su cuenta y comparar con la tabla 3.

TABLA 6

\begin{tabular}{|c|c|c|}
\hline Partido & Valor formal & Modificado \\
\hline PSE & 0,3667 & 0,3500 \\
\hline PNV ............... & 0,2667 & 0,3000 \\
\hline EA & 0,2667 & 0,3000 \\
\hline EE & 0,0333 & 0,0167 \\
\hline $\mathrm{CP}$ & 0,0333 & 0,0167 \\
\hline 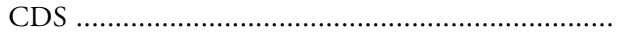 & 0,0333 & 0,0167 \\
\hline
\end{tabular}

Siguiendo el patrón empleado al estudiar el Parlamento al completo, la evaluación de las coaliciones ganadoras minimales del juego restringido y las preferencias de los partidos se presentan, respectivamente, en las tablas 7 y 8 .

\section{TABLA 7}

\begin{tabular}{|c|c|c|c|c|c|c|}
\hline & PSE & $P N V$ & $E A$ & $E E$ & $C P$ & $C D S$ \\
\hline Valor de Shapley .................. & 0,3500 & 0,3000 & 0,3000 & 0,0167 & 0,0167 & 0,0167 \\
\hline 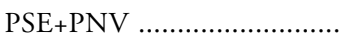 & 0,5250 & 0,4750 & & & & \\
\hline 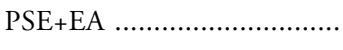 & 0,5250 & & 0,4750 & & & \\
\hline $\mathrm{PNV}+\mathrm{EA}+\mathrm{EE} \ldots \ldots \ldots \ldots \ldots \ldots \ldots$ & & 0,4583 & 0,4583 & 0,0833 & & \\
\hline$(\mathrm{PNV}+\mathrm{EA})+\mathrm{EE} \ldots \ldots \ldots \ldots \ldots \ldots$ & & 0,4375 & 0,4375 & 0,1250 & & \\
\hline $\mathrm{PNV}+(\mathrm{EA}+\mathrm{EE})$ & & 0,5000 & 0,4375 & 0,0625 & & \\
\hline$(\mathrm{PNV}+\mathrm{EE})+\mathrm{EA} \quad \ldots \ldots \ldots \ldots \ldots \ldots$ & & 0,4375 & 0,5000 & 0,0625 & & \\
\hline $\mathrm{PNV}+\mathrm{EA}+\mathrm{CP} \ldots \ldots \ldots \ldots \ldots \ldots \ldots \ldots$ & & 0,4583 & 0,4583 & & 0,0833 & \\
\hline$(\mathrm{PNV}+\mathrm{EA})+\mathrm{CP} \ldots \ldots \ldots \ldots \ldots \ldots \ldots$ & & 0,4375 & 0,4375 & & 0,1250 & \\
\hline $\mathrm{PNV}+(\mathrm{EA}+\mathrm{CP}) \ldots \ldots \ldots \ldots \ldots \ldots$ & & 0,5000 & 0,4375 & & 0,0625 & \\
\hline$(\mathrm{PNV}+\mathrm{CP})+\mathrm{EA} \ldots \ldots \ldots \ldots \ldots \ldots \ldots$ & & 0,4375 & 0,5000 & & 0,0625 & \\
\hline $\mathrm{PNV}+\mathrm{EA}+\mathrm{CDS} \ldots \ldots \ldots \ldots \ldots \ldots$ & & 0,4583 & 0,4583 & & & 0,0833 \\
\hline$(\mathrm{PNV}+\mathrm{EA})+\mathrm{CDS} \ldots \ldots \ldots \ldots \ldots$ & & 0,4375 & 0,4375 & & & 0,1250 \\
\hline $\mathrm{PNV}+(\mathrm{EA}+\mathrm{CDS}) \ldots \ldots \ldots \ldots \ldots$ & & 0,5000 & 0,4375 & & & 0,0625 \\
\hline$(\mathrm{PNV}+\mathrm{CDS})+\mathrm{EA} \ldots \ldots \ldots \ldots \ldots$ & & 0,4375 & 0,5000 & & & 0,0625 \\
\hline
\end{tabular}




\section{TABLA 8}

\begin{tabular}{lll}
\hline Partido & Coaliciones óptimas & Valor \\
\hline PSE & PSE+PNV & 0,5250 \\
& PSE+EA & 0,5250 \\
PNV & PNV+(EA+EE) & 0,5000 \\
& PNV+(EA+CP) & 0,5000 \\
& PNV+(EA+CDS) & 0,5000 \\
EA & (PNV+EE)+EA & 0,5000 \\
& (PNV+CP)+EA & 0,5000 \\
& (PNV+CDS)+EA & 0,5000 \\
EE & (PNV+EA)+EE & 0,1250 \\
CP & (PNV+EA)+CP & 0,1250 \\
CDS & (PNV+EA)+CDS & 0,1250 \\
\hline
\end{tabular}

Hay una diferencia esencial entre la situación que estamos considerando ahora y la que hemos estudiado antes, y es debida a la presencia de coaliciones binarias. Estas se forman de una única manera, y por tanto la cuestión estriba en saber si el PNV y EA, cada uno por su parte, prefieren la seguridad de un 0,4750 alineándose con el PSE o aspiran a obtener un 0,5000 entrando como tercer socio en una coalición ternaria pero arriesgándose a recibir menos o incluso a quedar fuera de las negociaciones por el entendimiento del otro con el PSE.

Nuestra selección se concreta aquí en las coaliciones

$$
\text { PSE+PNV, PSE+EA. }
$$

En definitiva y como conclusión, tras haber modificado la estructura formal del Parlamento del País vasco introduciendo restricciones a la cooperación derivadas de ciertas incompatibilidades binarias entre los partidos, el criterio de maximización del valor coalicional, que lleva implícito un criterio de estabilidad análogo al del equilibrio fuerte de Nash, ha conducido a seleccionar determinadas coaliciones como soluciones racionales al problema de la negociación:

a) Si todos los partidos acuden regularmente al Parlamento,

$$
\mathrm{PSE}+\mathrm{PNV}+\mathrm{CDS} \quad \mathrm{o} \quad \mathrm{PNV}+\mathrm{EA}+\mathrm{HB} .
$$

b) Si HB se mantiene sistemáticamente fuera del Parlamento,

$$
\mathrm{PSE}+\mathrm{PNV} \quad \mathrm{O} \quad \mathrm{PSE}+\mathrm{EA}
$$

Podemos comparar ahora estas conclusiones obtenidas in vitro con la actuación real de los partidos in vivo. Esta es la historia: durante los últimos días de 
enero - ya concluido este estudio-, EA y EE pactaron y ofrecieron su colaboración conjunta al PSE. Esto habría conducido a la coalición PSE+(EA+EE), que hemos descartado antes. Finalmente, el PSE no se integró, y EA+EE decidió presentar su candidatura a un gobierno minoritario. El 23 de febrero, el PSE y el PNV llegaron a un acuerdo. El pleno decisivo para elegir lehendakari había sido aplazado del 20 al 26 porque el gobierno español no daba permiso para salir de prisión a Juan Carlos Yoldi, candidato por HB.

Veintiséis de febrero de 1987: solamente dos candidatos. Yoldi por HB y Ardanza por PSE+PNV. Tras exponer su programa — un panegírico de ETA-, Yoldi y los demás parlamentarios de HB abandonaron la Cámara. José Antonio Ardanza no necesitó una segunda votación por mayoría simple: en la primera recibió los votos de PNV y PSE, naturalmente, y los votos del CDS.

La ausencia de HB permitió a la coalición PSE+PNV formar un gobierno de coalición que estuvo respaldado por 36 de los 62 votos del Parlamento. El apoyo del CDS garantizaba la mayoría absoluta en caso de que HB hubiera reaparecido en el Parlamento. Por otra parte, el reparto de competencias dentro del gobierno (aproximadamente al 50 por 100) también concuerda satisfactoriamente con los resultados proporcionados por nuestros cálculos del valor coalicional. Terminaba así el episodio iniciado el 30 de noviembre de 1986 cuando Ardanza, tras conocer que el PNV había sido superado claramente por el PSE, aseguraba que su partido "pasaba a la oposición», frase retórica que marcó el inicio de uno de los más largos, complejos y apasionantes períodos de negociación de nuestra historia reciente y que culminaría con la formación de una coalición «real».

Buena muestra de la estabilidad prevista por nuestro estudio es que la coalición PSE+PNV concluyó la Legislatura 1986-1990. En las cuartas elecciones vascas (1990), el PNV superó claramente al PSE por 22 a 16 escaños y se formó un gobierno de coalición tripartito $(\mathrm{PNV}+\mathrm{EE})+\mathrm{EA}$. A finales del verano del 91, tras haber pactado el PNV con el PSE después de las municipales de mayo, EA abandonó el gobierno y EE se escindió en dos formaciones: una tuvo corta vida y la otra, después de formarse un nuevo gobierno PNV+PSE+EE, acabó por integrarse en el PSE a finales del 92. En 1994, el PNV volvió a ganar al PSE, por 22 a 12 escaños en esta ocasión. Sin embargo, el PNV decidió establecer una política de coaliciones de escenario múltiple, ya que la peculiar estructura administrativa del País Vasco concede un papel muy importante al dominio de las Diputaciones Forales de las tres provincias vascas a través de sus Juntas Generales, cuya renovación había de producirse en mayo de 1995 junto con las elecciones municipales. Se formó la coalición $\mathrm{PNV}+\mathrm{PSE}+\mathrm{EA}$, y esas elecciones del 94 confirmaron la primacía del PNV - siempre sin llegar a la mayoría absoluta_, el desgaste socialista y el crecimiento del PP, frenado en parte por la segregación de UA en Alava. 


\section{CONCLUSIONES}

La teoría de los juegos cooperativos proporciona técnicas dotadas de la máxima racionalidad matemática para el análisis del comportamiento estratégico de los partidos en el marco parlamentario.

Los juegos de mayoría ponderada y, más en general, los juegos simples suministran los modelos adecuados para describir y estudiar los mecanismos de decisión colegiada típicos del ámbito político; el índice de Shapley-Shubik ofrece una medida de la distribución de poder a priori avalada por sus elegantes propiedades matemáticas.

El valor coalicional constituye una generalización plenamente coherente del índice de Shapley-Shubik que, conservando su significado y su sensibilidad, permite evaluar las alteraciones de poder debidas a la formación de coaliciones y dotar, por tanto, a los partidos de criterios objetivos para valorar los efectos de sus estrategias coalicionales, estableciendo un marco de negociación preciso cuyos resultados son de aplicación para racionalizar una ulterior distribución de cargos y áreas de responsabilidad en un eventual gobierno de coalición.

El criterio de optimización del valor coalicional conduce a la selección de determinadas coaliciones que, como valor añadido, gozan de una estabilidad análoga a la del equilibrio fuerte de Nash para juegos no cooperativos $y$, en consecuencia, aparecen como los resultados idóneos de la negociación intraparlamentaria.

Las implicaciones derivadas de la posición ideológica de los partidos, e incluso de sus conveniencias tácticas, admiten un tratamiento formalizado mediante su incorporación al modelo en términos de grafos de afinidad y/o incompatibilidad. Su incidencia queda reflejada en la sustitución del juego inicial por el juego modificado, que es susceptible de ser analizado mediante las técnicas ya descritas.

El estudio del Parlamento de Cataluña (1980-1984) y el Parlamento del País Vasco (1986-1990) ilustra adecuadamente la aplicación de las ideas anteriores, poniendo de manifiesto un notable grado de concordancia entre el comportamiento esperado desde la racionalidad matemática y el comportamiento real de los partidos en ambas situaciones.

\section{BIBLIOGRAFIA}

Amer, R., y Carreras, F. (1995a): "Games and cooperation indices», International Journal of Game Theory, 24, 239-258.

- (1995b): «Cooperation indices and coalitional value», Top, 3, 117-135.

AXELrod, R. (1970): Conflict of interest, Markham, Chicago.

Bergantiños, G.; Carreras, F., y García-Jurado, I. (1993): «Cooperation when some players are incompatible», en Operations Research, Games and Graphs (eds. S. Tijs y P. Borm), Methods and Models of Operations Research (ZOR), 38, 187-201. 
CAPO, J. (1986): «Party coalitions in the first democratic period in Spain, 1977-1982», en Coalitional Behavior in Theory and Practice: An Inductive Model for Western Europe (ed. G. Pridham), Cambridge University Press.

Carreras, F. (1991): "Restriction of simple games», Mathematical Social Sciences, 21, 245-260.

Carreras, F., y Owen, G. (1988): «Evaluation of the Catalonian Parliament, 1980-1984», Mathematical Social Sciences, 15, 87-92.

- (1995): «An analysis of the Euskarian Parliament, 1986-1990», en Social Choice and Political Economy (ed. N. Schofield), Kluwer, Boston, 261-273.

Carreras, F.; García-Jurado, I., y Pacios, M. (1993): «Estudio coalicional de los Parlamentos Autonómicos españoles de Régimen Común», Revista de Estudios Políticos, 82, 159-176.

Myerson, R. B. (1977): "Graphs and cooperation in games», Mathematics of Operations Research, 2, 225-229.

Owen, G. (1977): «Values of games with a priori unions», en Essays in Mathematical Economics and Game Theory (eds. R. Henn y O. Moeschlin), Springer-Verlag, 76-88.

- (1986): "Values of graph-restricted games», SIAM Journal of Algebraic and Discrete Methods, 7, 210-220.

Riker, W. H. (1962): The Theory of Political Coalitions, Yale University Press.

Shapley, L. S. (1953): "A value for n-person games", en Contributions to the Theory of Games (eds. H. W. Kuhn y A. W. Tucker), Princeton University Press, 307-317.

SHAPLEY, L. S., y SHUBiK, M. (1954): "A method for evaluating the distribution of power in a committee system", American Political Science Review, 48, 787-792.

Vázquez-Brage, M.; García-Jurado, I., y Carreras, F. (1995): «The Owen value applied to games with graph-restricted communication", Games and Economic Behavior (en prensa).

\section{RESUMEN}

Proponemos la aplicación del valor coalicional al estudio del comportamiento estratégico de los partidos en la formación de coaliciones parlamentarias. Del criterio de optimización del valor se deriva una noción de estabilidad equivalente a la del equilibrio fuerte de Nash para los juegos no cooperativos. Las restricciones a la cooperación por razones ideológicas o tácticas se incorporan al modelo formal. Como ilustración, analizamos el Parlamento de Cataluña (1980-1984) y el Parlamento del País Vasco (1986-1990).

\section{ABSTRACT}

This paper proposes the application of the coalitional value to the study of the strategic behaviour of political parties in terms of forming parliamentary coalitions. By capitalising on this value, a notion of stability is formed equivalent to Nash's strong equilibrium for non-cooperative games. The constraints to cooperation on ideological or tactical grounds are incorporated in the formal model. By way of illustration, both the Catalonian Parliament (1980$1984)$ and the Basque Parliament (1986-1990) are analysed. 